\title{
DESIGN OF A SENSOR-SUPPORTED MOBILE WORKING PLATFORM FOR ROUGH TERRAIN
}

\author{
Martin Zimmermann, Rolf Truninger, Gerhard Schweitzer \\ Institute of Robotics, ETH Zürich \\ CH-8092 Zürich, Switzerland \\ Martin Werder \\ siltec Verfahrenstechnik, Luggwegstr. 23 \\ CH-8048 Zürich, Switzerland
}

\begin{abstract}
New technologies and large financial investments will be required in the near future for the automation of forestry and construction work in the European mountainous regions, where hazardous work conditions strongly limit the access for conventional equipment. This paper presents the design of the roboTRAC, a man-driven sensor-supported mobile platform with on-board manipulator and intelligent tactile capabilities for mobility in rough terrain. The vehicle is based on a combined wheeled and legged configuration with a favourable weight to load ratio. The design of the lightweight hydraulically powered front legs with three degrees of freedom is presented. For supporting the operator in safely handling the complex walking apparatus a behavior-based control system is proposed, which provides a flexible framework for the implementation of different degrees of system autonomy. For interactive steering, the operator is provided with on-line static stability feedback in form of an equivalent reactive force, while a display is available for off-line planning of stable motions.
\end{abstract}

Keywords: mobile platform, legged locomotion, man-machine interface, intelligent interactive control, safety, stability

\section{INTRODUCTION}

New tools for the mechanisation and automation will be required for working in rough and mountainous terrain where the work conditions are hazardous and access for conventional equipment is strongly limited or impossible, thus making hard manual labor still indispensable today. As an example, large financial investments will be necessary in the European Alpine regions in the next decades for conservation work in forestry as well as for technical constructions for the protection of settlements, agricultural areas, traffic and energy installations from avalanches, landslips and floods. However, such maintenance and construction work has to be performed in a safe and economic way, while at the same time keeping damage to the work environment at a minimum. This requires lightweight, easily deployable equipment with sufficient mobility and safety features, thus opening a wide field of applications for new and unconventional mechanical configurations such as legged vehicles.

This paper presents the design of the roboTRAC, a man-driven sensor-supported mobile platform with on-board manipulator with intelligent tactile capabilities for mobility in extreme and rough terrain. As illustrated in figure 1, the vehicle is based on a combined wheeled and legged configuration specifically designed to allow for a favourable weight to load ratio, as the supporting wheels can carry much larger loads than active legs [1]. At the same time, the mechanical complexity as well as the costs are smaller for wheels than for legs. With the partially folded legs used as a tractor hitch, the roboTRAC may also be trailed by a motor tractor on roads. In addition, with its front legs with three degrees of freedom each and its active rear wheels, the platform offers sufficient mobility to allow for traversing rough terrain and adapting well to any work site. The platform also offers inherent safety features, as it is 
impossible to lift a leg when the vehicle is not statically stable. Unlike mechanically similar configurations with hinged support front legs which are already in use in Europe, built for example by Schaef Company, Germany, Menzi, Switzerland, or Kaser, Austria, the maneuverability is strongly improved, and important safety and operator support features are added. Furthernors, the roborkAC is capable of maneuvering in any enain independent of the tool it is carrying, thereby expanding the range of applications of suct existing vehicles. Potential tools comprise various types of excavating ams, rock-drilling ecuipment or feller buncher processing units as already applied in forestry work today. The practical objectives of the roboTRAC configuration and application examples are discussed in more detail in [2].

The roboTRAC project is promoted by the Swiss Commission for the Promotion of Scientific Research and has been accepted by the European Community as EUREKA project No. 322. Several small Swiss companies are participating in the project, being mainly concerned with the planning of processes in agriculture and forestry (siltec Verfahrenstechnik) and with control and engineering aspects (Sägesser Engineering, Celsi AG). Research is done at the Institute of Robotics of the ETH Zürich, and some investigations have been performed in cooperation with the University of Duisburg, Germany, [3], and the University of Arizona, USA [4].

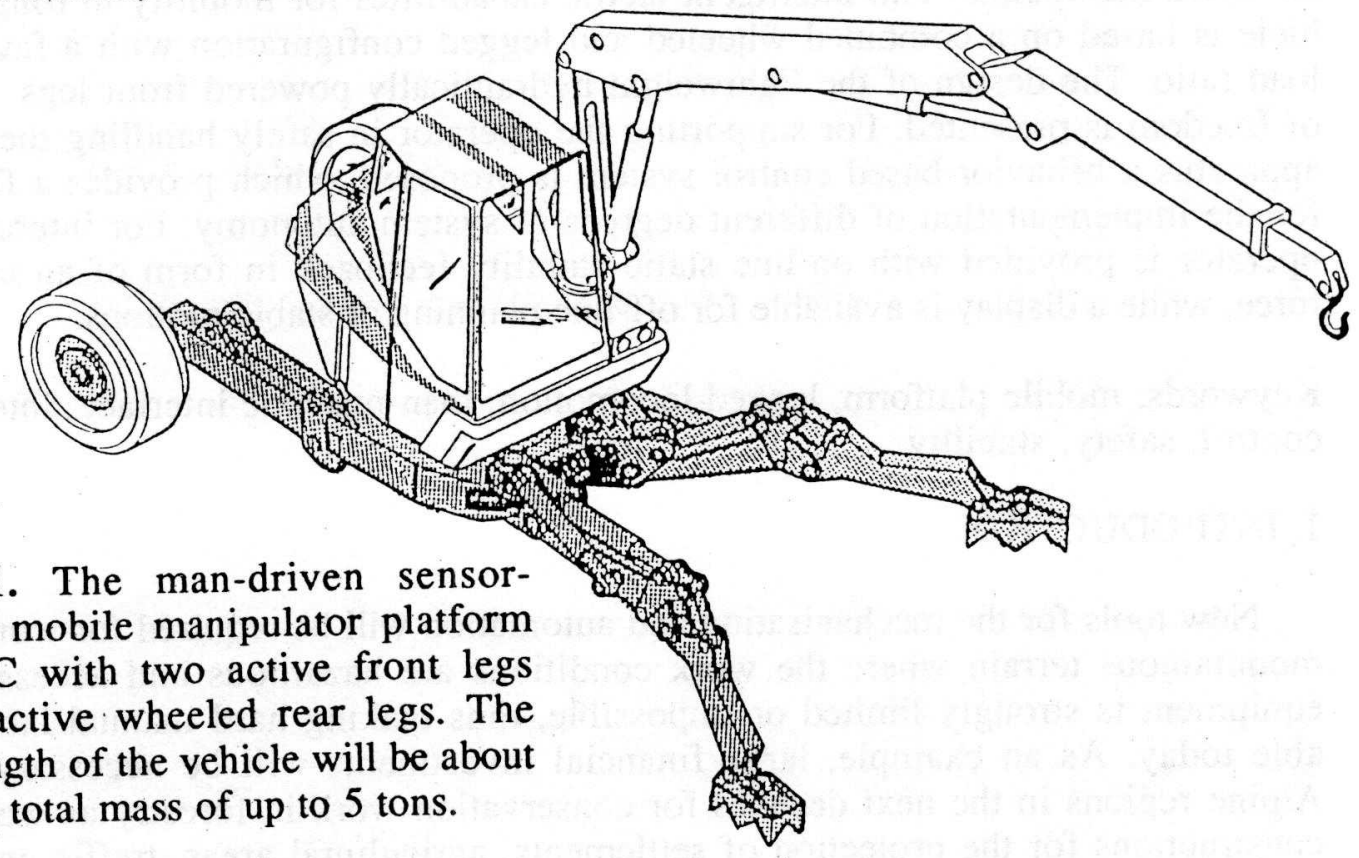

\section{DESIGN REQUIREMENTS}

In order to support and enhance the capabilities of the human operator and finally lead to increased efficiency in work performance and a widened range of applications, the roboTRAC must behave as an intelligent, interactive machine. The automatic control and the sensory system must relieve the operator from the repetitive and exhaustive tasks mainly associated with handling the complex mechanism in order to allow him to concentrate on performing the actual task at hand. Given this overall objective and the mechanical configuration with two front legs and two rear wheels, all design requirements for a wide range of practical applications of the roboTRAC under varying environmental conditions have been listed, including requirements stemming from an analysis of the human operator interactions, safety features, implementation and testing issues, as well as maintenance considerations:

- The human operator must have an efficient and ergonomically designed communication system available, thus effectively allowing to drastically reduce the training periods of the 
human operators. In particular, the roboTRAC must be kept stable and the state of the static stability be monitored and fed back to the human operator at all times.

- The human operator should be able to issue high-level commands and choose between various operating modes best suited to the task at hand. He must be relieved from steering and coordinating single actuators whereever feasible and plausible, thereby reducing fatigue during operation.

- The roboTRAC should be able to operate safely at various levels of autonomy in any type of terrain. In particular, it must be capable of autonomously performing leg coordination and safe foothold determination on various types of terrain and surfaces.

- The mechanical structure should be lightweight with a favourable load to weight ratio, allowing for reasonable payloads while keeping ground pressure at a minimum and the vehicle even being possibly deployable by helicopter. Its maximum speed in open terrain should reach up to $4 \mathrm{~km} / \mathrm{h}$.

- The vehicle must allow for good maintainability and debuggability. It must be possible to gradually enhance its capabilities by building up the control system incrementally, at the same time leading to graceful degradation properties.

\section{MECHANICAL DESIGN}

Following the list of the major design requirements provided in the last section, several demands have been derived for the mechanical design of a prototype model. The basic mechanical design of the central body, as well as that of the two wheeled rear legs and the on-board manipulator, can be taken from the mechanically similar configurations already in use. However, the front leg, had to be newly designed. For the prototype front leg, the following specifications have been formulated:

- Three degrees of freedom to be actuated by three actuators;

- Large work range with appropriate geometry that is reachable without encountering kinematical singularities, thus allowing for good stability and high mobility;

- Possibility to completely fold the upper and lower limb for easy transport on or behind a truck and small-sized, safe storage of the roboTRAC;

- Ability to apply the necessary forces of up to 20 to $30 \mathrm{kN}$;

- A minimum velocity of about $1.5 \mathrm{~m} / \mathrm{s}$ given the desired forces and power resources;

- Robustness with regard to the planned applications in forestry and construction;

- Suitable costs to be achieved by using standard off-the-shelf equipment wherever possible.

\subsection{Design of the Front Leg of the roboTRAC}

In order to find a design solution that is best suited to meet these requirements, several different configurations and actuation principles have been studied. Most existing walking machines such as the adaptive suspension vehicle [5], employ a pantograph mechanism as shown in figure 2a. It offers low power consumption for a quasi static gate and linear foot movements when a single actuator is moved. However, the goal of the roboTRAC project is to build a robust platform with high mobility in rough terrain, while maximum energy efficiency is not of major importance. Hence, the disadvantage of the pantograph leg, are valued higher. They include that the manufacturing of the sledges for prismatic joints is far more expensive than that of simple revolute joints. In addition, this design may lead to problems with toler- 
ances and robustness in harsh and dirty environments. Furthermore, the work range of a pantograph mechanism is worse when compared to other kinematic configurations (see figure 2). As a result, among five other configurations, the parallelogramm mechanism shown in figure $2 \mathrm{~b}$ has been chosen for the front leg of the roboTRAC. It is compact, robust and provides sufficient energy efficiency and a suitable work range. Attached to the parallelogram mechanism, the foot always keeps the same orientation as the central body. The leg will be powered by linear hydraulic cylinders, since the needs for high power density as well as the demands on robustness exclude any other actuation principle.

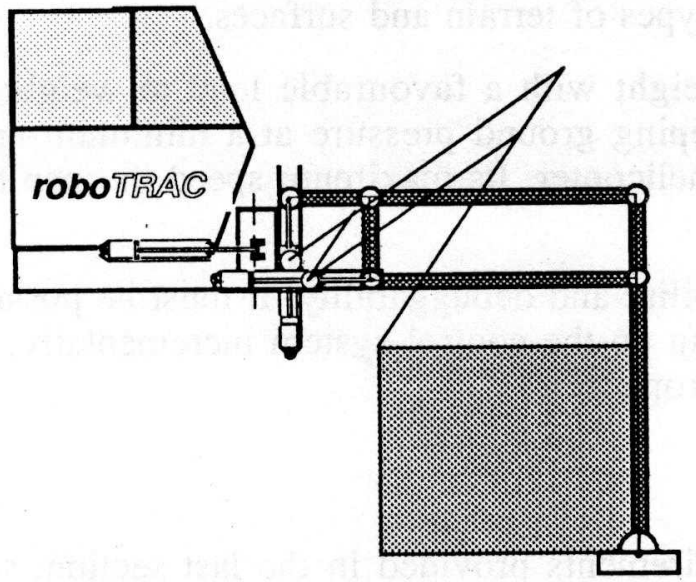

Figure 2a. Pantograph mechanism.

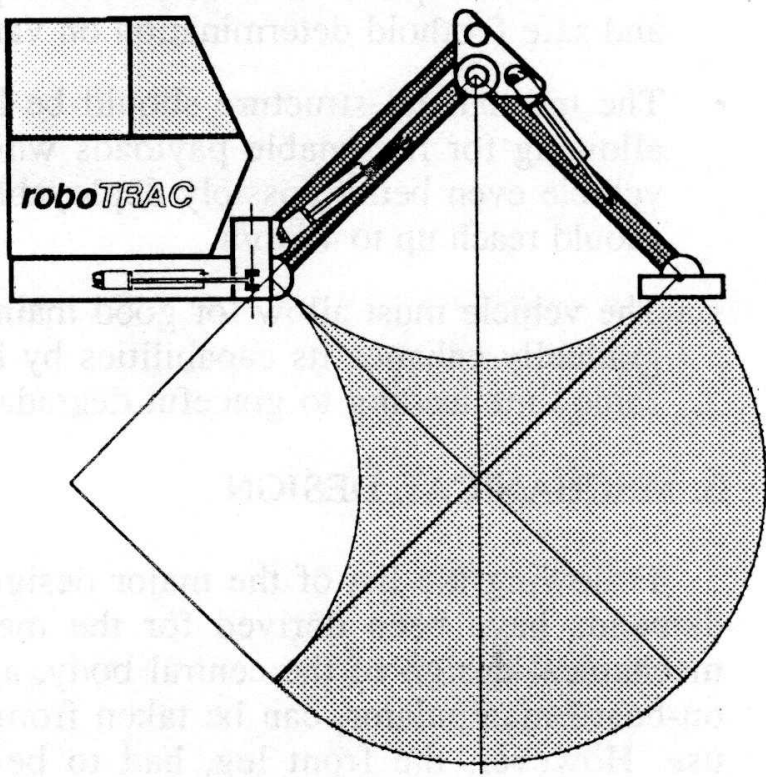

Figure 2b. Parallelogramm mechanism.

Figure 2. Comparison of the geometry and work space of a pantograph mechanism and the parallelogramm mechanism finally chosen for the roboTRAC prototype leg. For both mechanisms, the orientation of the foot relative to the platform body is always maintained.

\subsection{Characteristics of the Prototype under Construction}

To serve as a testbed for the roboTRAC, the prototype must be capable of carrying a man, the sensors and the electronic hardware. A scale of 1:2 proved to fulfill these demands, in particular since standard hydraulic actuators can be used. The prototype leg now being built consists of three hydraulic actuators with integrated position and pressure sensors as well as servovalves. Its total mass will be about $100 \mathrm{~kg}$. The leg can apply forces up to 5000 $\mathrm{N}$ in any direction with a maximum speed of $0.8 \mathrm{~m} / \mathrm{s}$ at a supply pressure of the hydraulic system of $18 \mathrm{MPa}$. The system pressure is chosen relatively low in order to have some reserves available when the prototype model is scaled up to its original size.

\section{SYSTEM ENGINEERING}

\subsection{The Basic Operating Modes of roboTRAC}

In order to achieve the overall objective of building a mobile manipulator system with a widened range of applications, the entire system has to be designed to enhance and support human operator capabilities rather than just imitate or replace them. As a first consequence, 
this demands that the human operator be actively embedded in the information household of the system, requiring that the command and the information feedback pathways be chosen appropriately. However, this leaves the division of the tasks between the human operator and the automatic control system as the major task for system engineering, which needs to be decided before actually designing the control system or the man-machine interface. This section deals with this division, while issues concerning the specific controller design and information feedback are left to sections 5 and 6.

As experienced in other applications of supervisory control systems (such as in space robotics, see for example, [6]), automatic control systems are best suited to cope with highbandwidth actions that generally require little intelligence such as repetitive or fine motions, while tasks with lower bandwidth generally involve higher intelligence and are at best left to a human operator who is supported by sensors and a control system. However, depending on the particular task at hand, the system must be flexible enough so as to provide for the appropriate level of system autonomy while still ensuring sufficient ability for operator interactions.

For the roboTRAC, the different operating modes and command variables have been chosen based on a study of the ergonomics of the set of tasks to be performed. As a result, three basic operator-selectable operating modes will be available for the roboTRAC:

- Walking mode: this mode will be used when the platform has to be transferred to a new location in any type of terrain. The platform is steered by the operator with operatorselectable varying degrees of autonomy. Generally, the on-board manipulator will not be moved during this operation;

- Repositioning mode: once the vehicle has reached its work site, its legs have to be positioned as to allow for maximum platform stability, safety, and tool reachability with respect to the work task at hand;

- Working mode: when the platform has been positioned appropriately for the given task, the platform actuators will be locked in place and the desired task is to be performed with the on-board manipulator.

\subsection{Flexible Operation within the Basic Operating Modes}

Within the basic operating modes, the operator may still choose between different levels of autonomy of the machine. All commands will be given by two three-axis joysticks operated with the left and right hand, respectively, and the total number of major operator-controlled variables is limited to six. The command directions are chosen as to cause a resulting action in the same direction as the movement of the command lever. In addition, the commanded variables are selected to allow for ready understanding and interpretation of the resulting actions, thus effectively reducing the burden of performing complex input-output mappings from the operator which is essential for reducing the training periods. However, the safety supervision system will be active independent of the selected operating mode, allowing the operator to concentrate on the few most important variables during the specific task at hand.

As an example, two submodes will be available during walking mode. Obviously, depending on the type of terrain to be traversed and the particular task, the operator may either decide to concentrate on navigation and path-planning while leaving leg coordination and foothold selection to the automatic controller, or to control leg motion and foot placement independently of the automatic controller. The latter mode may be employed for close maneuvering or in very rough and unsafe terrain, while higher autonomy like automatic walking may be desired when maximum travel speed in less problematic terrain is more important. 


\section{DESIGN OF THE MAN-MACHINE INTERFACE}

\subsection{Man-Machine Interactions}

The potential loss of the static stability poses a major safety hazard for all mobile manipulators such as swing cranes or the roboTRAC. Accidents caused by overturning often cause great destruction of equipment and even loss of lives. A major reason for those accidents is that the static stability is not continuously monitored and the operator not informed about the state of stability. Indeed, stability is a rather abstract kind of information which cannot be sensed or easily learned by human operators. The objective of this section is to present a man-machine interface capable of communicating the abstract information about the static stability of the platform to the operator in an effective and readily understandable manner during all phases of the operation. The proposed interface enables the human operator to safely perform the task at hand without interference from stability monitoring tasks.

The analysis of the operating modes employed by human operators revealed, that a human operator roughly plans movements or sequences thereof initially. In a second phase, the planned movements will likely be executed with some on-line modifications due to initial planning uncertainties. However, those modifications may put the stability at risk, thus possibly requiring further modifications or cancelation of the desired movements. Hence, computer assistance is inevitable during both operating modes in order to enhance the safety and the work performance. The state of the static stability is computed based on algorithms provided by [4], where the static stability is defined in terms of the value of the virtual power needed to overturn the vehicle about a potential axis of rotation.

\subsection{The Ergonomic Design of the Man-Machine Interface}

For planning larger movements or sequences thereof, an off-line stability monitoring system is proposed. The system consists of a display, showing a projected view of the platform and the manipulator along with all contact points to the ground. Superposed to this projection are the various zones of allowable positions of the manipulator within its maximum work range. The zones are determined as a function of the load to be carried by the manipulator. Different shadings or colours are employed to achieve good readability. The interpretability is further enhanced by keeping the projection of the cabin fixed and letting the zones of stability rotate during manipulator movements, thereby also maintaining the viewpoint of the operator. This can be further enhanced by employing an overhead display rather than a front panel-based display.

In addition, the static stability must be monitored and fed back to the operator continuously. The three main human communication channels have been compared under these requirements: acoustic feedback, visual feedback, and mechanical feedback, which could be realized by forces exerted onto the control device. Compared to mechanical feedback, acoustic and particularly visual feedback prove rather slow for human beings. In a possibly noisy environment, acoustic feedback can also be easily overheard. Since the actual manipulation task will be controlled by visual feedback, this channel is already busy. Thus, any additional visual information may be overlooked by the operator who has to concentrate his efforts on the manipulation task at hand. In addition, visual as well as acoustic feedback involve rather complex and difficult to learn input-command mappings, requiring long training periods. On the other hand, mechanical feedback effectively removes those perceptual problems by allowing to shift the computational burden mainly to the control computer. Moreover, multidirectional feedback becomes possible. Hence, mechanical feedback is chosen as the preferrable channel to communicate on-line stability information to the operator 
of the roboTRAC. However, on-line stability feedback is by no means restricted to legged vehicles but will be generally applicable to any type of wheeled or legged platform [7].

\subsection{Implementation of the On-line Stability Feedback}

The on-line stability feedback is implemented by using an active force-controlled lever. Figure 3 shows a possible hardware configuration. Electric motors induce forces into the joystick which are measured by a force sensor and fed back to the motor control unit. The position of the device, as measured by the encoders of the motors, is then used to command the movement of a leg or the manipulator. The sensors used to evaluate the actual margin of static stability depend on the known variables of the mechanical model. If the mass geometry is known, position sensors for the joint coordinates and pitch and roll inclination sensors are the minimal equipment. These sensors are complemented by force sensors at the contact points of the legs, for example, in order to get additional information about the load conditions at the contact points as well as some redundancy.

Figure 3. The currently evaluated hardware configuration of a two-axis force-controlled control lever. Electric motors and the operator induce forces at the lever which are measured by a force sensor and fed back to the motor control unit.

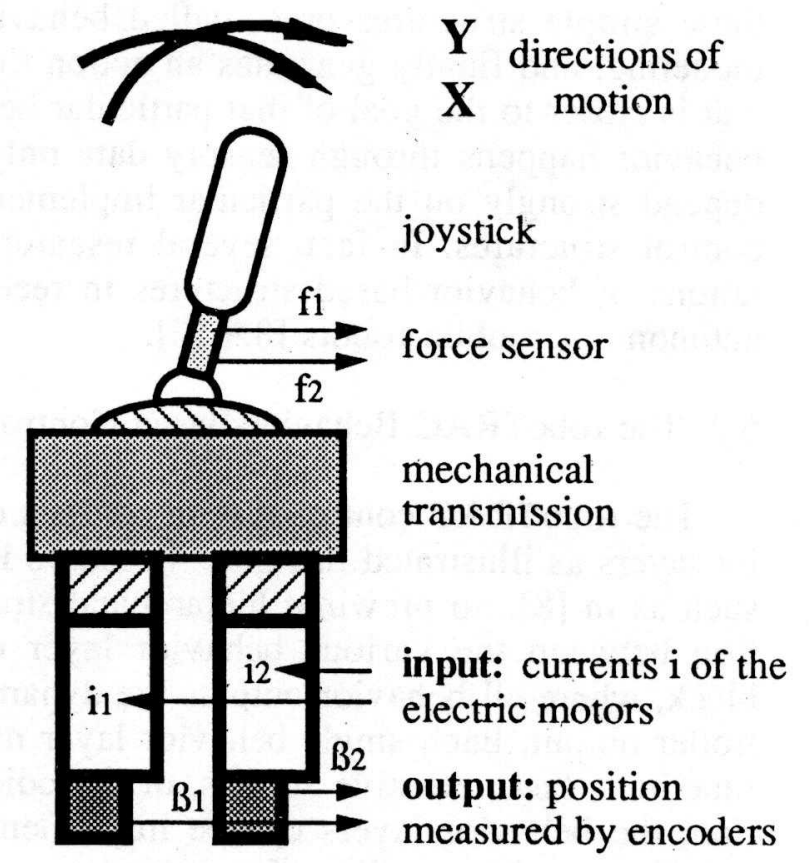

The mapping between the static stability measure and the reactive force is rather complex. Various physiological aspects must be considered. The demands below have been derived:

- The magnitude of the reactive force is equivalent to the margin of static stability and its direction is determined by the negative gradient of static stability;

- When the margin of static stability decreases to zero, the forces in the corresponding degree of freedom of the joystick reach their maximum value, independent of the magnitude of the control command;

- The forces also depend on the position of the joystick. Control commands of large magnitude undergo stronger opposition forces than those of small magnitude.

- All control movements have to be done actively. A certain hold off force protects the operator from submitting commands unintentionally. 


\section{CONTROL SYSTEM DESIGN}

\subsection{Design Requirements and Controller Selection}

The automatic control system of the roboTRAC supports the human operator as to relieve him from the task of handling the complex mechanical apparatus of the roboTRAC, thus allowing the operator to concentrate entirely on performing the task at hand. In particular, the control system must be designed to meet the system engineering demands as discussed in Section 4.

For fulfilling these complex requirements, a behavior-based control system has been designed. Behavior-based control structures are rooted in the control schemes observed in biological systems, the basic underlying idea being that the overall system behavior may be achieved by a collection of rather simple but simultaneously operating structures which are competing at the command level [8]. Hence, rather than having a functional decomposition of tasks, such as central sensor data acquisition, central data fusion, perception, modeling, etc., task decomposition is based on the various subgoals that have to be achieved. Thus, each of these simple structures or so-called behaviors acquires its own data, does perception and modeling, and finally generates an action that is intended to push the system towards a state that is closer to the goal of that particular behavior. The communication between the layers of behavior happens through sensory data only. The resulting properties of the overall system depend strongly on the particular implementation within this framework of behavior-based control structures. In fact, several researchers have already developed specific implementations of behavior-based structures in recent years, mostly for applications in the field of autonomous mobile robots $[8,9,10]$.

\subsection{The roboTRAC Behavior-Based Control System}

The roboTRAC control system consists of multiple completely independent simple behavior layers as illustrated in figure 4. Unlike in most other behavior controller implementations such as in [8], no prewired hierarchical structure exists within the system. Instead, competition between the various behavior layer outputs happens in a separate command fusion block, where all behavior outputs are dynamically weighted and summed up to form the controller output. Each single behavior layer may perform controller tasks, monitoring or safety functions, fast, reflexive actions, or periodic and sequential actions. Independent of its function, the behavior layers can be implemented as classical controllers, logical operators, or neural network controllers, for example.

This implementation leads to a control system for the roboTRAC which exhibits the required characteristics:

- Incremental buildup and testing can be done due to the distributed organization of knowledge, finally allowing also graceful degradation;

- Action-oriented perception is achieved, since each behavior acquires and processes only the sensory data required for its particular subtask and finally competes with the other subtasks;

- The competition of outputs at the command level offers the capability for the resolution of conflicts between various subtasks;

- The execution of outdated commands is unlikely since the processing time per behavior layer can be kept small and communication through the world happens fast; 
- Most important, an automatic control layer may also be replaced by a human operator function, thus allowing high flexibility in choosing the degree of autonomy and operator interactivity.

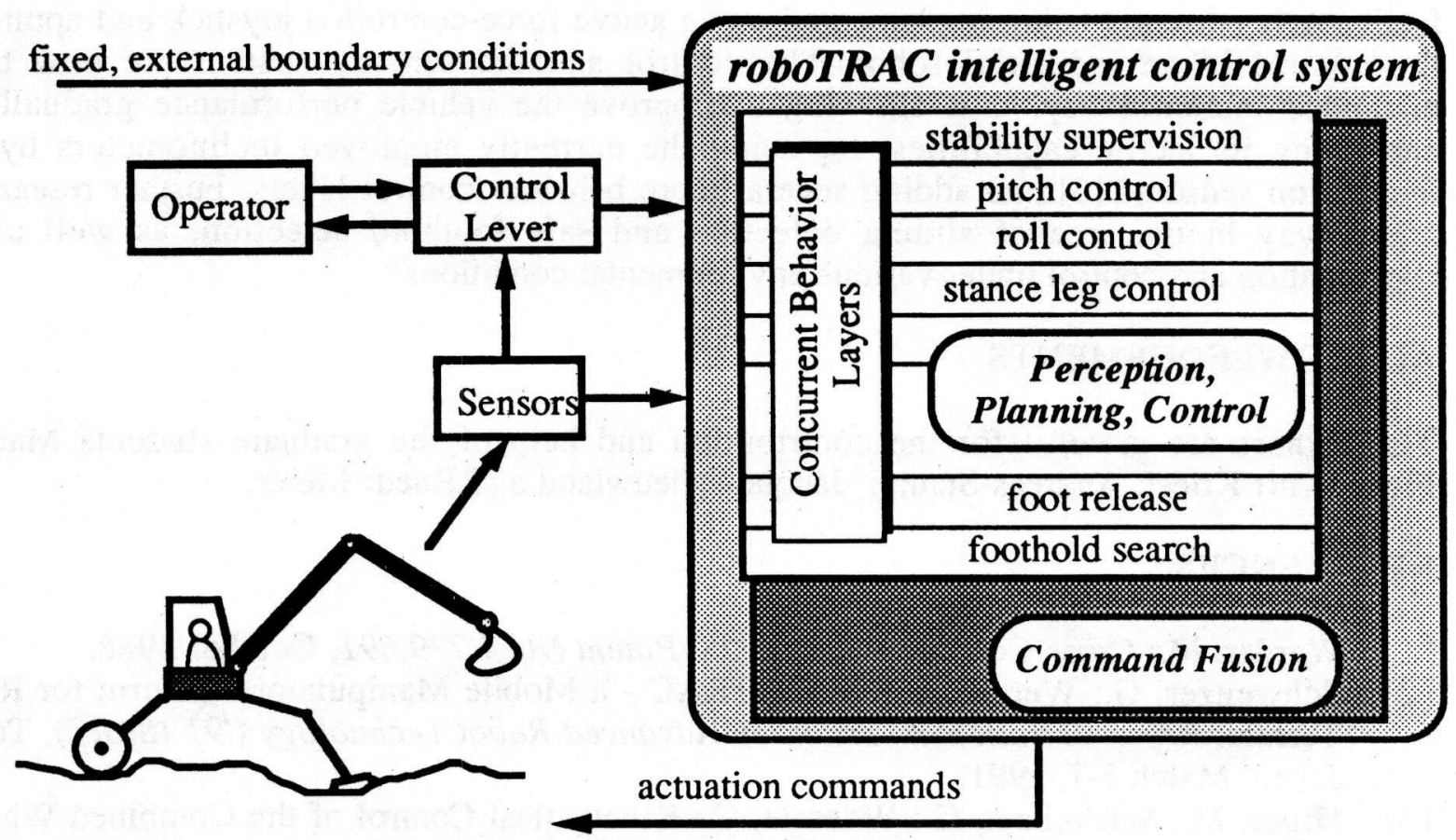

Figur 4. The behavior-based control system of the roboTRAC. The human operator issues commands by using an active control lever, which are then possibly accepted and executed, modified or rejected by the intelligent control system. Several of the behavior control layers are shown. Competition between the various behavior layers happens in a separate command fusion block.

\subsection{Experimental Implementation}

The control system for the automatic walking mode has been implemented at a scaleddown version of the roboTRAC. All computations are done by an off-board VME-bus computer system with a Motorola 68020 Processor with mathematical coprocessor running the real-time multiprocessor operating system XMETH developed at the Institute of Robotics. All software is written in MODULA-2. The model is equipped with tactile contact sensors at all four legs, joint angle sensors, and a two-axis inclinometer. Walking is performed by combining the stance leg motion and a series of reflexes carried out by the swing leg, following observations from biologists obtained from locusts and stick insects $[11,12]$. No traditional leg motion and foot placement planning is performed. All layers have been designed independently.

With the implemented behavior layers, the vehicle is capable of traversing rough terrain while keeping the platform in a horizontal position, as long as the swinging leg does not hit an obstacle during the forward swing phase. In particular, the system already possesses some very essential inherent safety features. For example, static stability during walking is considerably improved by controlling the platform roll angle, and the vehicle stops when no foothold is detected, thus effectively avoiding to fall into deep ditches. 


\section{OUTLOOK}

The presented design of the man-driven sensor-supported manipulator system roboTRAC with intelligent tactile capabilities has been shown to allow for high mobility in rough and extreme terrain while guaranteeing the stability of the vehicle at all times. The on-line feedback is currently being implemented on an active force-controlled joystick and applied to the control of an industrial robot. The control and the sensory system are now being expanded incrementally, thus allowing to improve the vehicle performance gradually by enhancing its tactile capabilities, replacing the currently employed inclinometers by fast inclination sensors [13] and adding several more behavior control layers. Further research is under way in the area of sliding detection and safe foothold selection, as well as leg coordination and control under various environmental conditions.

\section{ACKNOWLEDGEMENTS}

The authors are grateful for the contribution and help of the graduate students Matthias Flury, Kurt Kriesi, Andreas Staubli, Jacques Nieuwland and Ruedi Meier.

\section{REFERENCES}

[1] Werder, M.: Cross Country Vehicle, US. Patent No. 4,779,691, October 1988.

[2] Schweitzer, G.; Werder, M.: ROBOTRAC - a Mobile Manipulator Platform for Rough Terrain, Proc. '91 Intl. Symposium on Advanced Robot Technology ('91 ISART), Tokyo, Japan, March 5-7, 1991.

[3] Hiller, M.; Schweitzer, G.; Woernle, C.: Kinematical Control of the Combined Wheeled and Legged Vehicle ROBOTRAC, to be published in the Proc. of the 8th CISMIFToMM Symp. RO.MAN.SY'90, Cracow, Poland, July 1990.

[4] Davidson, J.K.; Schweitzer, G.: A Mechanics-Based Computer Algorithm for Displaying the Margin of Static Stability in Four-Legged Vehicles, ASME Jrl. of Mechanical Design, Vol. 112, December 1990.

[5] Hong, S.M.; Waldron, K.J.: Machines That Walk: The Adaptive Suspension Vehicle, MIT Press, Cambridge, MA, USA, 1989.

[6] Heindl, J.; Hirzinger, G.; Landzettel,K.: Sensorgeführte Roboter mit neuen MenschMaschine Schnittstellen, Technical Report, Deutsche Forschungsanstalt für Luft- und Raumfahrt (DLR), Oberpfaffenhofen, Germany, 1989.

[7] Truninger, R.; Werder, M.; Zimmermann, M.: Zentralkörper mit einem Bedienelement, CH-Patentgesuch Nr. 620/91-0, March 1991.

[8] Brooks, R.: A Robust Layered Control System for a Mobile Robot, IEEE Jrl. of Robotics and Automation, Vol. RA-2, No. 1, 1986.

[9] Arkin, R.C.: Motor Schema Based Navigation for a Mobile Robot, IEEE Conf. on Robotics and Automation, Rayleigh, NC, USA, March 1987.

[10] Anderson, T.L.; Donath, M.: A Computational Structure for Enforcing Reactive Behavior in a Mobile Robot, SPIE Vol. 1007 Mobile Robots III, 1988.

[11] Bässler, U.: Neural Basis of Elementary Behavior in Stick Insects, Springer Verlag, Berlin, Heidelberg, New York, 1983.

[12] Pearson, K.G.; Franklin, R.: Characteristics of Leg Movements and Patterns of Coordination in Locusts Walking on Rough Terrain, The Intl. Jrl. of Robotics Research, Special Issue on Legged Locomotion, Vol. 3, No. 2, Summer 1984.

[13] Zimmermann, M.; Sulzer, W.: High Bandwith Orientation Measurement and Control Based on Complementary Filtering, submitted for presentation at the IFAC Symposium on Robot Control (SYROCO'91), Vienna, Austria, September 16-18, 1991. 